\title{
Analysis of repair of replication-born double-strand breaks by sister chromatid recombination in yeast
}

\author{
Belén Gómez-González, Pedro Ortega, and Andrés Aguilera* \\ Centro Andaluz de Biología Molecular y Medicina Regenerativa (CABIMER), Universidad de \\ Sevilla-CSIC-Universidad Pablo de Olavide, Seville, Spain \\ *Corresponding author: e-mail address: aguilo@us.es
}

\section{Contents}

1. Introduction 122

1.1 The study of DSB repair mechanisms 123

$\begin{array}{ll}1.2 \text { Repair of broken replication forks } & 124\end{array}$

2. The use of a mini-HO site to study SCR induced by replication-born DSBs generated by the $\mathrm{HO}$ endonuclease

3. The use of the FRT site and a mutant FLP to study replication-born

DSB-induced SCR

4. Rationale of the TINV recombination systems 126

5. Reagents, materials and equipment 129

$\begin{array}{ll}5.1 \text { Reagents } & 129\end{array}$

5.2 Materials 130

$\begin{array}{ll}5.3 \text { Equipment } & 131\end{array}$

6. Protocols 131

6.1 Replication-born DSB induction 131

6.2 Genetic determination of recombination frequencies 132

6.3 Physical analysis of DSBs and SCR intermediates 132

7. Data analysis 136

Acknowledgments 136

References 136

\section{Abstract}

The repair of DNA double-strand breaks is crucial for cell viability and the maintenance of genome integrity. When present, the intact sister chromatid is used as the preferred repair template to restore the genetic information by homologous recombination. Although the study of the factors involved in sister chromatid recombination is hampered by the fact that both sister chromatids are indistinguishable, genetic and 
molecular systems based on DNA repeats have been developed to overcome this problem. In particular, the use of site-specific nucleases capable of inducing DNA nicks that replication converts into double-strand breaks has enabled the specific study of the repair of such replication-born double strand breaks by sister chromatid recombination. In this chapter, we describe detailed protocols for determining the efficiency and kinetics of this recombination reaction as well as for the genetic quantification of recombination products.

\section{Introduction}

Double-strand breaks (DSBs) are among the most cytotoxic DNA lesions as a single unrepaired DSB is potentially lethal. Consequently, multiple options have evolved in eukaryotic cells to ensure that DSBs are repaired. However, not all possible DSB repair pathways result in the same outcome. Whereas some repair pathways accurately restore the DNA without consequences, the processing and repair of DSBs can also moderately or even extensively alter the genetic information, leading to mutations, loss of heterozygosity, or chromosome rearrangements that could ultimately result in cell death or tumorigenesis. Hence, to ensure accurate DSB repair is crucial for the maintenance of genome integrity. Despite that exogenous and endogenous agents can access and harm the DNA leading to various lesions including DSBs, one major source of genome instability is related to the DNA metabolism itself. Among DNA metabolic processes, evidence indicates that replication has a strong potential to cause alterations in the DNA, being $S$ phase the most vulnerable phase of the cell cycle. Spontaneous DSBs occurring during DNA replication must therefore be carefully repaired to warrant fidelity. In accordance, the major repair pathway used during $\mathrm{S}$ phase is Sister Chromatid Recombination (SCR), a recombination reaction in which the genetic information from the intact sister chromatid is used as a template to restore the broken DNA (Johnson \& Jasin, 2000; Kadyk \& Hartwell, 1992).

Here, we describe the tools and methodology developed and used for the study of the repair of DSBs generated during DNA replication thanks to the use of site-specific nucleases that are able to produce nicks in the DNA before replication converts them into DSBs in the budding yeast Saccharomyces cerevisiae, vastly used as an eukaryotic model organism to study DNA DSB repair due to its easy growth and genetic manipulation. 


\subsection{The study of DSB repair mechanisms}

There are two major DSB repair pathways: non-homologous DNA end joining (NHEJ), which re-ligates the two DNA ends together, and homologous recombination (HR), which uses the genetic information found in an homologous DNA sequence as a template to repair the break, resulting in either the unidirectional transfer of information (gene conversion) from the template (donor) to the broken molecule (recipient) or a reciprocal exchange of information between both molecules or crossover (reviewed in Pardo, Gómez-González, \& Aguilera, 2009; Heyer, Ehmsen, \& Liu, 2010). NHEJ can potentially ligate all kinds of double-stranded DNA ends and it is in large part mutagenic, in contrast to error-free HR. Indeed, HR restores the information of the broken molecule without altering its DNA sequence when using an identical copy as template, as is the case of a sister chromatid. This explains that, when available (in the S and G2 phases of the cell-cycle), the sister chromatid is the preferred donor molecule (GonzalezBarrera, Cortes-Ledesma, Wellinger, \& Aguilera, 2003; Johnson \& Jasin, 2000; Kadyk \& Hartwell, 1992). Notwithstanding, the donor used for the HR repair reaction can be in the same locus of the homologous chromosome, leading to allelic recombination, or in any other location, leading to ectopic recombination. Thus, ectopic homologous DNA sequences or repeats, either in a different or the same DNA molecule whether a chromosome or a plasmid, can be used as alternative templates for the repair of DSBs. Apart from point mutations or loss of heterozygosity (LOH) as possible readouts of allelic recombination, ectopic recombination between DNA repeats can lead to important chromosomal reorganizations like deletions, duplications, inversions and translocations.

Diverse genetic systems based on DNA repeats have been developed and extensively used for the study of DSB repair by the different HR mechanisms: Single-Strand Annealing (SSA), Synthesis-Dependent Strand Annealing (SDSA), Double-Strand Break Repair (DSBR), and Break-Induced Replication (BIR) (Gómez-González, Ruiz, \& Aguilera, 2011; Klein et al., 2019). Thus, constructs based on a duplication of non-identical tandemrepeats have been highly useful for the study of SCR. Despite the fact that the two sister chromatids resulting from replication are genetically indistinguishable, unequal SCR events occurring between the tandem-repeats and leading to triplications of the repeat can be genetically distinguishable. This rationale was used in the first yeast genetic assay generated to study 
spontaneous SCR (Fasullo \& Davis, 1987), and has been used later applied to study DSB-induced SCR in yeast (Fasullo, Bennett, \& Dave, 1999) and human cells (Johnson \& Jasin, 2000; Puget, Knowlton, \& Scully, 2005). However, an additional layer of complexity is required to physically study SCR and its kinetics of occurrence during time-course experiments. This was achieved with plasmid systems carrying either one ( $p$ L2-HOr system) or two ( $p$ TINV system) copies of the LEU2 gene. In these cases, recombination intermediates from equal or unequal SCR events leading to reciprocal exchanges between the sister chromatids (Sister Chromatid Exchange, SCE) could be detected by Southern-blot after DNA-break induction by a site-specific endonuclease (Gonzalez-Barrera et al., 2003). Importantly, the studies with those systems support that unequal SCE accurately represents a reliable readout of natural SCR (Gonzalez-Barrera et al., 2003).

\subsection{Repair of broken replication forks}

During replication, advancing forks can encounter different sorts of obstacles that might cause their stalling or blockage (reviewed in Gómez-González \& Aguilera, 2019). Specific protein-induced replication fork (RF) barriers have been used to study the fate of RFs after blockage (Lambert \& Carr, 2013; Willis \& Scully, 2021). Upon stalling, if replication does not resume, forks can collapse and break. This would be the case of a fork encountering a nick in the template. As a result, a one-ended DSB would be generated that could promote replication restart via BIR (see Anand, Lovett, \& Haber, 2013; Yeeles, Poli, Marians, \& Pasero, 2013 for reviews). However, once an incoming RF arrives from the opposite side, a two-ended DSB would be generated resembling a standard two-ended DSB. The proximity of the sister chromatids, which are held by cohesin rings, promotes such replication-born DSBs to be preferentially repaired with the sister chromatid (Cortés-Ledesma \& Aguilera, 2006), thus maximizing the chances of restoring the genetic information without harmful consequences.

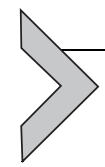

\section{The use of a mini-HO site to study SCR induced by replication-born DSBs generated by the $\mathrm{HO}$ endonuclease}

The yeast homothallic (HO) switching endonuclease is a site-specific endonuclease responsible for mating type switching that works by introducing a nick in both DNA strands and thus leading to a 4-bp staggered DSB into a 117 bp-target sequence (HO site) (Rudin, Sugarman, \& Haber, 1989). 
The $\mathrm{HO}$ endonuclease is involved in the cleavage of the yeast $M A T$ locus in chromosome III. However, the expression of the $\mathrm{HO}$ endonuclease in a strain carrying the MATa-inc mutation, with a non-cleavable mutated HO site (Mascioli \& Haber, 1980) allows the use of the HO site to induce DSBs elsewhere to study its repair without interference by mating type switching. Interestingly, the use of a mini-HO target site of $24 \mathrm{bp}$ (mini$\mathrm{HO}$ ) lowers the efficiency of the $\mathrm{HO}$ endonuclease at that site (GonzalezBarrera et al., 2003). In this case, a large fraction of DSBs induced at this 24-bp mini-HO site would be replication-born as they would occur when the RF hit the nicks randomly generated in either DNA strand (CortésLedesma \& Aguilera, 2006). It is worth mentioning that the mini-HO site can also be occasionally cleaved in both strands at the same time causing replication-independent DSBs, although this happens in a low frequency (less than 2\%) (Cortés-Ledesma \& Aguilera, 2006).

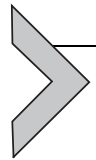

\section{The use of the FRT site and a mutant FLP to study replication-born DSB-induced SCR}

The Flippase (FLP) endonuclease of the yeast $2 \mu$ circle plasmid is involved in site-specific recombination induced by single-stranded cleavage and ligation reactions at the two 48-bp FLP recognition target (FRT) sites of the $2 \mu$ circle (Sadowski, 1995). FLP recombinase action at the FRT leads to an intermediate FLP cleavage complex (FLP-cc), which is rapidly resolved by the ligation step (Lee, Whang, \& Jayaram, 1996). However, a first step-arrest mutant version of FLP (FLPm) in which the histidine at position 305 is substituted by leucine (H305L), causes an irreversibly FLPm protein-bound nick that cannot be ligated (Tsalik \& Gartenberg, 1998) similar to the TOP1cc formed after treatment with the DNA topoisomerase I poison camptothecin. Thus, the FLPm nicking enzyme can be used to introduce site-specific nicks at the FRT site that after replication would result in DSBs (Nielsen et al., 2009). However, in contrast to those produced by the $\mathrm{HO}$ endonuclease at the mini-HO site, FLPm-induced nicks are strand-specific. This allows, for instance, to target the nicks to the replication leading or lagging strands (Ortega, Gómez-González, \& Aguilera, 2019). Furthermore, the frequency at which FLPm cleaves both strands is below $0.2 \%, 10$-fold lower than that of the $\mathrm{HO}$ at the mini-HO site, making this a much better nickase with lower background levels of DSB induction (Ortega et al., 2019). 


\section{Rationale of the TINV recombination systems}

The TINV recombination systems are based on a centromeric plasmid carrying the $U R A 3$ gene as the selective genetic marker and two inverted repeats of the LEU2 gene carrying different mutations (Gonzalez-Barrera et al., 2003; Ortega et al., 2019). One of the LEU2 copies is $5^{\prime}$-truncated whereas the other one is a complete gene interrupted by the mini-HO sequences (TINV-HO system) or FRT (TINV-FRT system), which are inserted at the LEU2 EcoRI site (leu2-HOr or leu2-FRT alleles, respectively) (Fig. 1A) (Gonzalez-Barrera et al., 2003). These systems allow both the genetic detection of Leu + recombination products (Fig. 1A) that, although a good indirect indicator of unequal SCR products, can also arise via other HR events, as well as the physical detection of unequal SCR intermediates (Fig. 1B-D) preferentially arising from the SCE between the cut leu2-HOr repeat in one chromatid and the truncated leu $2 \Delta 5^{\prime}$ repeat in the sister chromatid.

To study SCR, the endogenous LEU2 gene must be deleted in the strain of interest to avoid ectopic recombination and undesired probe hybridization during physical analysis by Southern-blot (Gonzalez-Barrera et al., 2003). In the case of the TINV-HO, the yeast strain must also be $M A T a-i n c$ to avoid HO cleavage at the $M A T$ loci. The expression of the $\mathrm{HO}$ or FLPm endonucleases must be inducible, which is achieved by placing the corresponding genes under the GAL promoter. GAL-HO and GALFLPm constructs are available to be directly expressed from the genome in ade3::GAL-HO strains (Gonzalez-Barrera et al., 2003) or in HPHMX6:: GAL-FLPm yeast strains (Ortega et al., 2019), respectively. Alternatively, the GAL-HO and GAL-FLPm constructs are also available in centromeric plasmids with different selective genetic markers to be co-transformed with the pTINV plasmid of interest (pTINV-HO or pTINV-FRT): pRS413GALHO (HIS3) (Muñoz-Galván, Jimeno, Rothstein, \& Aguilera, 2013), pBISGALkFLP (TRP1) and pBIS-GALkFLP (URA3) (Tsalik \& Gartenberg, 1998). Additionally, the GAL-HO construct has been inserted together with the TINV recombination system in the same centromeric plasmid allowing to perform experiments with single transformants: pTHGH (URA3) and pTHGH-2 (TRP1) (Ortega et al., 2019).

Time-course experiments are performed after HO or FLPm induction to determine the kinetics of DSB formation and HR repair. Southern-blot hybridization with a LEU2 specific probe after XhoI-SpeI digestion allows 
A

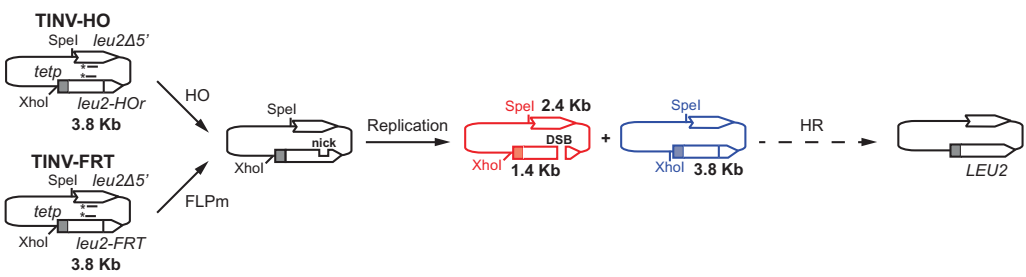

B

Sister Chromatid Recombination (SCR)
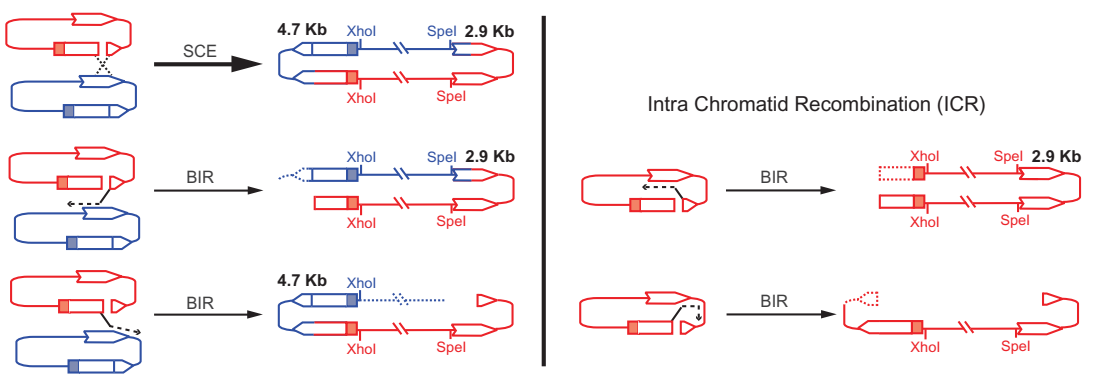

C

TINV-HO

TINV-FRT

Gal (h): $0 \begin{array}{llllllllllllll}1 & 1.5 & 3 & 4.5 & 6 & 7.5 & 9 & 0 & 1.5 & 3 & 4.5 & 6 & 7.5 & 9\end{array}$
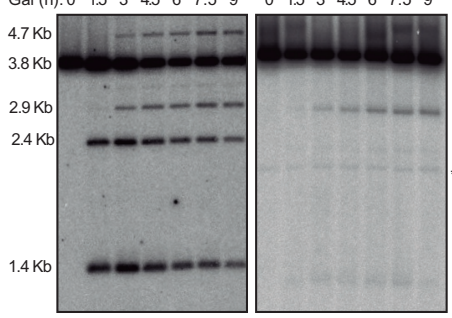

D

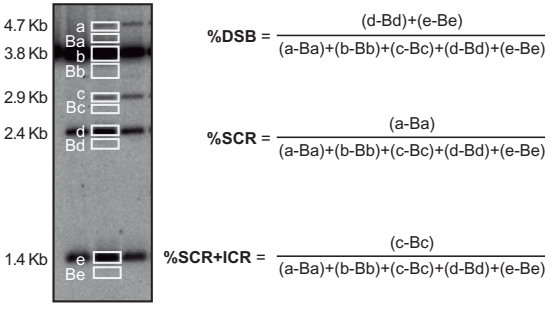

E
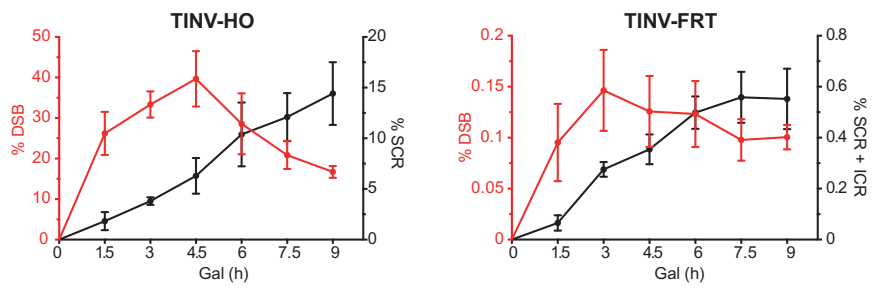

Fig. 1 Analysis of repair of replication-born DSBs with TINV plasmid systems. (A) Scheme of the TINV-based recombination plasmid systems (TINV-HO and TINV-FRT) with two inverted repeats of the LEU2 gene, one $5^{\prime}$ truncated (leu $2 \Delta 5^{\prime}$ ) and the other one placed under the doxycycline-repressible promoter (tetp) and interrupted by the mini-HO or FRT sequences (leu2-HOr or leu2-FRT alleles, respectively). Induction of the expression of either $\mathrm{HO}$ or FLPm endonucleases creates a nick that is converted into a DSB in one of the chromatids (red) by replication, while the sister chromatid (blue) remains intact. All Xhol and Spel restriction sites are indicated.

(Continued) 
the visualization of the molecules of interest (Fig. 1C). A 3.8-Kb band corresponding to the linearized plasmid is observed in all time points. Break induction leads to 2.4- and 1.4-kb bands (Fig. 1A and C). At longer times, new 4.7- and 2.9-kb bands appear that indicate the occurrence of HR. This can happen by SCR using as a template either of the leu 2 copies located in the sister chromatid (equal SCR with the leu2-HOr/leu2-FRT or unequal SCR with the leu $\triangle \triangle 5^{\prime}$ ) or by Intra-Chromatid Recombination (ICR) using as a template the leu $2 \Delta 5^{\prime}$ copy located within the same chromatid (Fig. 1B). Unequal SCR leads to the formation of recombination intermediates that correspond to the 4.7- and 2.9-kb bands generated after the XhoI-SpeI digestion. Importantly, the $4.7-\mathrm{kb}$ band is specifically generated by SCR, and it is known to occur mostly by unequal SCE, with BIR being only a minor contributor (Cortes-Ledesma, Tous, \& Aguilera, 2007). In contrast, the 2.9-kb band results also from either sister-chromatid BIR or

Fig. 1-Cont'd Fragments generated after DSB induction and Xhol-Spel digestion, as detected by the LEU2 probe shown as a line with asterisk are indicated with their corresponding sizes. HR repair can give rise to restoration of the LEU2 gene Leu + recombinants. (B) Scheme of different recombination intermediates generated by either unequal Sister Chromatid Recombination (SCR), which can occur by Sister Chromatid Exchange (SCE) or by Break-Induced Replication (BIR) with the leu $\triangle \triangle 5^{\prime}$ copy in the intact sister chromatid, or by Intra-Chromatid Recombination (ICR) by BIR with the leu $\triangle 5^{\prime}$ copy within the same chromatid. The size of fragments generated after DSB induction and Xhol-Spel digestion, as detected by the LEU2 probe (shown in Fig. $1 \mathrm{~A}$ ) are indicated. Dashed lines depict putative fragments of undefined length depending on the extent of DNA synthesis of the BIR reaction. (C) Kinetics of appearance of DSBs and HR repair intermediates with the TINV-HO and TINV-FRT systems as determined by Southern blot hybridization with the LEU2 probe depicted in Fig. 1A. The different-sized molecules are generated after Xhol-Spel digestion and either HO (TINV-HO system) or FLPm (TINV-FRT system) induction in galactose for the indicated time-points. Bands resulting from unspecific hybridization are indicated with an asterisk. These illustrative gels correspond to experiments published in Ortega et al. (2019). (D) Diagram for the quantification of the percentage of each kind of molecule (DSBs, SCR intermediates or SCR + ICR intermediates). The signal from same-sized quantification squares over each band of interest ( $a, b, c$ or $d)$, from which the corresponding Background (B) signal must be subtracted, is divided by the sum of the signal of each all other bands. (E) Results of the mean and SEM values from the quantification of the bands obtained in a wild-type strain transformed with from either pTINV-HO or pTINV-FRT plasmids. The percentage of molecules with DSBs is shown in red in both systems whereas the percentage of molecules that constitute an intermediate for HR reactions is shown in black. SCR intermediates were quantified in the TINV-HO system and SCR+ICR intermediates in the TINV-FRT system. The data correspond to independent experiments published in Ortega et al. (2019). 
Intra-Chromatid Recombination (ICR) occurring between the two inverted repeats located within the broken chromatid (Fig. 1B), both reactions known to occur at low frequency (Cortes-Ledesma et al., 2007; Cortés-Ledesma \& Aguilera, 2006; Gonzalez-Barrera et al., 2003). Quantifying the amount of the different-sized DNA molecules allows to infer the efficiency and kinetics of each HR reaction (Fig. 1D and E).

\section{Reagents, materials and equipment}

\subsection{Reagents}

- Glucose-containing minimal media (SC): $1.7 \mathrm{~g} / \mathrm{L}$ amino acid-free yeast nitrogen base, $5 \mathrm{~g} / \mathrm{L}$ ammonium sulfate, $20 \mathrm{~g} / \mathrm{L}$ glucose. Add $20 \mathrm{~g} / \mathrm{L}$ agar before autoclaving for solid media. Autoclave and supplement with the required amino acid. All amino acids should be added in standard concentrations except for leucine and adenine, which should be added three times more concentrated

- Raffinose-containing minimal media (SRaf): $1.7 \mathrm{~g} / \mathrm{L}$ amino acid-free yeast nitrogen base, $5 \mathrm{~g} / \mathrm{L}$ ammonium sulfate. Autoclave and supplement with the required amino acid. All amino acids should be added in standard concentrations except for leucine and adenine, which should be added three times more concentrated and with $2 \%$ raffinose (Thermo Fisher Scientific, cat \# 15815188) from a 20\% autoclaved stock solution

- 20\% galactose (Sigma-Aldrich, cat \# G0750) filtered stock solution

- $5 \mathrm{mg} / \mathrm{mL}$ doxycycline stock (Sigma-Aldrich, cat \# D9891). Store in the dark at $4{ }^{\circ} \mathrm{C}$ for up to 2 weeks

- $1 \mathrm{M}$ spermidine stock solution (Sigma-Aldrich, cat \# S2626). Store at $-20^{\circ} \mathrm{C}$. Make a fresh stock solution of this reagent every month

- 0.5 M spermine stock solution (Sigma-Aldrich, cat \# S3256). Store at $-20^{\circ} \mathrm{C}$

- Nuclei-isolating buffer (NIB) pH 7.2: 17\% (w/v) glycerol, $50 \mathrm{mM}$ (3-[N-morpholino] propanesulfonic acid) sodium salt (MOPS, SigmaAldrich, cat \# M1254) pH 7.5, $150 \mathrm{mM} \mathrm{CH}{ }_{3} \mathrm{CO}_{2} \mathrm{~K}$ (Sigma-Aldrich, cat \# P1190), $2 \mathrm{mM} \mathrm{MgCl} 2,500 \mu \mathrm{M}$ spermidine, $150 \mu \mathrm{M}$ spermine. Autoclave and store at $4{ }^{\circ} \mathrm{C}$ in the dark for up to 6 months

- Zymolyase 20T (15 mg/mL) (USBiological)

- RNase A (10 mg/mL) (Sigma-Aldrich, cat \# R6513)

- $3 \mathrm{M}$ sodium acetate

- Phenol solution (Sigma-Aldrich, cat \# P4557) 
- Phenol:Chloroform:Isoamyl alcohol solution (25:24:1) (Sigma-Aldrich, cat \# P3803)

- Isopropanol

- Milli-Q water

$-1 \times$ TE: Tris-HCl $10 \mathrm{mM}$, EDTA $1 \mathrm{mM} \mathrm{pH} 8$

- $100 \%$ ethanol

- Ethidium bromide (10 mg/mL) (Sigma-Aldrich, cat \# E1510)

- Agarose

- $1 \times$ TBE: $90 \mathrm{mM}$ Tris base, $90 \mathrm{mM}$ boric acid, $2 \mathrm{mM}$ EDTA

- 1-kb DNA ladder (Invitrogen, cat \# 10787018 or similar)

- $10 \times$ Loading buffer

- Xhol and SpeI-HF restriction enzymes (New England Biolabs, cat \# R0146L and R3133L, respectively)

$-0.25 \mathrm{M} \mathrm{HCl}$

- Denaturation solution: $0.5 \mathrm{M} \mathrm{NaOH}, 1.5 \mathrm{M} \mathrm{NaCl}$

- $20 \times$ SSC: $0.3 \mathrm{M} \mathrm{NaCl}, 0.03 \mathrm{M}$ trisodium citrate $\mathrm{pH} 7$

- dATG solution: $0.5 \mathrm{mM}$ dATP, $0.5 \mathrm{mM}$ dTTP, and $0.5 \mathrm{mM}$ dGTP

- Specific LEU2 oligonucleotides (5'-GTTCCACTTCCAGATGAGGC$3^{\prime}$ and $5^{\prime}$-TTAGCAAATTGTGGCTTGA-3')

- $\alpha 32^{\mathrm{P}}$-dCTP $(1 \mathrm{mCi}[10 \mathrm{mCi} / \mathrm{mL}], 3000 \mathrm{Ci} / \mathrm{mmol})$ (PerkinElmer, cat \# NEG513H250UC)

- Klenow (Roche, cat \# 1008412001)

- Hexanucleotide mix (Roche, cat \# 11277081001)

- Taq DNA polymerase (MyTaq ${ }^{\mathrm{TM}}$ DNA polymerase) (Bioline, cat \# BIO-21105)

- NucleoSpin Gel and PCR extraction kits (Macherey-Nagel, cat \# 740609.50)

- Sephadex G50-TE (GE Healthcare, cat \# 11524875): Dissolve $5 \mathrm{~g}$ Sephadex G50 in $75 \mathrm{~mL} 1 \times \mathrm{TE}$, autoclave, and store at $4{ }^{\circ} \mathrm{C}$

- Hybridization solution: $0.5 \mathrm{M}$ phosphate buffer $\mathrm{pH} 7,7 \% \mathrm{SDS}$

- Wash solution: $0.1 \times$ SSPE, $5 \mathrm{mM}$ EDTA, 0.5\% SDS

- $20 \times$ SSPE: $3 \mathrm{M} \mathrm{NaCl}, 200 \mathrm{mM}$ sodium phosphate, $20 \mathrm{mM}$ EDTA $\mathrm{pH} 7.4$

\subsection{Materials}

- $50 \mathrm{~mL}$ tubes

- $1.5 \mathrm{~mL}$ microtubes

$-2 \mathrm{~mL}$ microtubes 
- Fluorometer cuvettes

- Temperature controlled shaker

- Temperature controlled incubator

- Phase lock gel (PLG) heavy $2 \mathrm{~mL}$ tubes (Quantabio, cat \# 2302830)

- Hybond-XL hybridization nylon membrane (GE-Healthcare, cat \# RPN303S)

- Whatman filter paper

- Filter paper

- Petri dishes

- Fluorescent gel ruler

\subsection{Equipment}

- Centrifuge for $50 \mathrm{~mL}$ tubes

- Centrifuge for $1.5 \mathrm{~mL}$ tubes

- Fluorometer apparatus

- Block heater or water bath

- Magnetic stirrer

- Electrophoresis apparatus

- Electrophoresis power supply

- UV transilluminator

- 254 nm UV crosslinker

- Hybridization oven

- Hybridization bottles

- Vortex

- Nanodrop

- PhosphorImager Fujifilm FLA-5100 (Fujifilm)

- ImageGauge software (Fujifilm)

\section{Protocols}

\subsection{Replication-born DSB induction}

1. Zig-zag streak the yeast transformant of interest on an SC plasmid-selective media plate for 3-4days at the selected temperature $\left(30^{\circ} \mathrm{C}\right.$ for non-thermosensitive strains).

2. Inoculate a single colony in $5 \mathrm{~mL}$ liquid $\mathrm{SC}$ plasmid-selective media and incubate overnight on a shaker at the appropriate temperature $\left(30^{\circ} \mathrm{C}\right.$ for non-thermosensitive strains). 
3. Dilute the culture in $50 \mathrm{~mL}$ of the same SC plasmid-selective media and let it grow for two rounds of duplication. This usually takes around $6 \mathrm{~h}$. Collect the cultures at OD600 of 0.6-0.8, before lag phase.

4. Dilute the culture in $350 \mathrm{~mL}$ for a of SRaf plasmid-selective media and incubate overnight at the appropriate temperature.

Tip: To avoid any putative interference of transcription from the TET promoter placed in the leu2 repeat to be cut (leu2-HOr or leu2FRT), add doxycycline to a final concentration of $5 \mu \mathrm{g} / \mathrm{mL}$.

Tip: For the genetic determination of recombination frequencies, steps 2-4 can be avoided and the different colonies of the yeast transformant of interest can be directly inoculated in $5 \mathrm{~mL}$ SRaf plasmidselective media with doxycycline $(5 \mu \mathrm{g} / \mathrm{mL})$ if possible. Make sure to use colonies of the same size to be able to assume that the frequencies are estimated from the same number of cell divisions.

5. Wait until OD600 of 0.4-0.5, add galactose to a final concentration of $2 \%$ and keep shaking to induce the expression of either the HO or FLPm endonuclease.

6. The time of incubation will depend on the goal of the experiment (genetic determination of recombination frequencies or physical analysis of DSBs and SCR intermediates), as explained below.

\subsection{Genetic determination of recombination frequencies}

To determine recombination frequencies, galactose-induction should be kept during $5 \mathrm{~h}$ for $\mathrm{HO}$ and $6 \mathrm{~h}$ for FLPm. After that, add glucose to a final concentration of $2 \%$ to stop the endonuclease expression and perform serial dilutions in $\mathrm{H}_{2} \mathrm{O}$ to be plated on either SC plasmid-selective media, from which we can estimate the total number of cells; or SC plasmid-selective media lacking leucine, from which we can estimate the number of recombinant cells.

\subsection{Physical analysis of DSBs and SCR intermediates}

To determine the kinetics of DSB formation and repair by HR, the plasmid DNA is extracted at different time points after galactose addition (usually 0 , $1.5,3,4.5,6,7.5$ and $9 \mathrm{~h}$ ). At every time-point, harvest $50 \mathrm{~mL}$ of the cell culture in $50 \mathrm{~mL}$ tubes, centrifuge at $4{ }^{\circ} \mathrm{C}(3000 \mathrm{~g}, 2 \mathrm{~min})$, remove the supernatant and wash the pellet with cold milli-Q $\mathrm{H}_{2} \mathrm{O}$. Centrifuge again $(3000 \mathrm{~g}$, $2 \mathrm{~min}$ ), remove the supernatant and freeze each pellet at $-80^{\circ} \mathrm{C}$. In addition, take $1 \mathrm{~mL}$ of the cell culture for measuring OD in the fluorometer apparatus with the appropriate cuvettes. Write down the ODs for every time-point as 
these values will be the reference to estimate the volume containing the same number of cells at every time-point. Once all time-point samples are collected, proceed with the adjusted volumes as follows:

\subsubsection{DNA extraction}

1. Add $1 \mathrm{~mL}$ of milli- $\mathrm{Q} \mathrm{H}_{2} \mathrm{O}$ to the frozen pellet and transfer the previously estimated volume of cells into a $2 \mathrm{~mL}$ microtube, centrifuge $(3000 \mathrm{~g}, 1 \mathrm{~min})$, remove supernatant and resuspend the pellet in $400 \mu \mathrm{L}$ NIB by vortexing.

2. For cell wall digestion, add $80 \mu \mathrm{L}$ of zymolyase $20 \mathrm{~T}(15 \mathrm{mg} / \mathrm{mL})$ and incubate during $35 \mathrm{~min}$ at RT inverting the tubes every $10 \mathrm{~min}$.

3. To stop the zymolyase action, add $1.5 \mathrm{~mL}$ of milli-Q $\mathrm{H}_{2} \mathrm{O}$ to each tube, centrifuge ( $3000 \mathrm{~g}, 2 \mathrm{~min}$ ), remove supernatant and resuspend the pellet in $720 \mu \mathrm{L}$ of $1 \times$ TE by pipetting and a subsequent vigorous vortexing. From now on, do not use the vortex anymore.

4. For cell lysis, add $80 \mu \mathrm{L} 10 \%$ SDS and incubate on ice during $30 \mathrm{~min}$ inverting the tubes every $10 \mathrm{~min}$ during the incubation.

5. For protein removal, add $800 \mu \mathrm{L}$ phenol, mix vigorously, centrifuge $(16,000 \mathrm{~g}, 15 \mathrm{~min})$ and transfer the clear upper phase into a $2 \mathrm{~mL}$ microtube. Add $800 \mu \mathrm{L}$ Phenol:Chloroform:Isoamyl alcohol solution, mix vigorously, centrifuge $(16,000 \mathrm{~g}, 5 \mathrm{~min})$ and transfer the clear upper phase into a new $2 \mathrm{~mL}$ PLG heavy tube. Repeat this last step at least once more in order to obtain a clean sample.

6. For nucleic acid precipitation, add $160 \mu \mathrm{L}$ sodium acetate $3 \mathrm{M}$ and $800 \mu \mathrm{L}$ isopropanol, invert the tubes several times, centrifuge $(16,000 \mathrm{~g}, 15 \mathrm{~min})$ and remove supernatant. Add $700 \mu \mathrm{L}$ of cold $70 \%$ ethanol without mixing, centrifuge $(16,000 \mathrm{~g}, 10 \mathrm{~min})$, remove supernatant and resuspend the pellet in $500 \mu \mathrm{L} 1 \times \mathrm{TE}$.

7. To remove RNA, add $0.5 \mu \mathrm{L}$ RNase $\mathrm{A}(10 \mathrm{mg} / \mathrm{mL})$, resuspend the pellet and incubate at $37^{\circ} \mathrm{C}$ during $30-60 \mathrm{~min}$.

8. To remove RNAse A, add $500 \mu \mathrm{L}$ Phenol:Chloroform:Isoamyl alcohol solution and transfer both phases into a $2 \mathrm{~mL}$ PLG heavy tube, mix vigorously, centrifuge $(16,000 \mathrm{~g}, 5 \mathrm{~min})$ and transfer the clear upper phase into a new $2 \mathrm{~mL}$ microtube.

9. Precipitate the DNA by adding $100 \mu \mathrm{L}$ sodium acetate $3 \mathrm{M}$ and $500 \mu \mathrm{L}$ isopropanol. Centrifuge $(16,000 \mathrm{~g}, 15 \mathrm{~min})$ and remove supernatant. Add $700 \mu \mathrm{L}$ of cold $70 \%$ ethanol without mixing, centrifuge $(16,000 \mathrm{~g}, 10 \mathrm{~min})$, remove supernatant and resuspend the pellet in $200 \mu \mathrm{L} 1 \times$ TE. 
10. Quantify the DNA in each preparation with a DNA fluorimeter and use either $5 \mu \mathrm{g}$ or $10-20 \mu \mathrm{g}$ of DNA, for HO and FLPm inductions, respectively, for digestion with $50 \mathrm{U}$ of $\mathrm{XhoI}$ and SpeI-HF restriction enzymes $\left(2 \mathrm{~h}\right.$ at $\left.37^{\circ} \mathrm{C}\right)$.

11. Precipitate the digested DNA by adding $100 \mu \mathrm{L}$ sodium acetate $3 \mathrm{M}$ and $500 \mu \mathrm{L}$ isopropanol. Invert the tubes several times, centrifuge $(16,000 \mathrm{~g}, 15 \mathrm{~min})$ and remove supernatant. Add $700 \mu \mathrm{L}$ of cold $70 \%$ ethanol without mixing, centrifuge $(16,000 \mathrm{~g}, 10 \mathrm{~min})$, remove supernatant and resuspend the pellet in $30 \mu \mathrm{L} 1 \times \mathrm{TE}$.

Tip: Make sure to let the pellet dry before dissolving in $1 \times \mathrm{TE}$ avoiding water and/or ethanol rests.

Tip: If the extracted DNA pellet is not properly dissolved, use pre-warmed the $1 \times \mathrm{TE}$ and incubate the pellet in it for $30-60 \mathrm{~min}$ at $30^{\circ} \mathrm{C}$.

\subsubsection{Gel electrophoresis}

12. Prepare a $0.8 \%$ agarose gel by dissolving $2.8 \mathrm{~g}$ agarose in $350 \mathrm{~mL}$ boiling $1 \times \mathrm{TBE}$ with a stir bar in a magnetic stirrer. Let it cool down to $65^{\circ} \mathrm{C}$ in a water bath or a temperature-controlled incubator. Add ethidium bromide (to a $0.3 \mu \mathrm{g} / \mathrm{mL}$ final concentration) and then pour the solution in a $24 \times 20 \mathrm{~cm}$ gel-casting tray to allow agarose polymerization (usually during $2 \mathrm{~h}$ ). Then, place the gel at a gel stage containing a suitable volume of $1 \times \mathrm{TBE}$ and clean the wells with a syringe or a pipette.

Tip: The agarose must be perfectly diluted in order to avoid non-dissolved agar clumps.

13. Load $5 \mu \mathrm{L}$ of a $1-\mathrm{kb}$ DNA ladder in a well and the digested DNA samples $(30 \mu \mathrm{L}$ with $3.3 \mu \mathrm{L}$ of $10 \times$ Loading buffer each) in the rest of the wells. Run at constant low voltage $(50 \mathrm{~V}$, c.a. $1 \mathrm{~V} / \mathrm{cm})$ overnight. Stop the electrophoresis when the 1-kb band from the 1-kb DNA ladder reaches the end of the gel (usually after $20 \mathrm{~h}$ ).

14. Take a picture of the ethidium bromide staining in a UV transilluminator with a fluorescent gel ruler.

\subsubsection{Transfer of DNA to a nylon membrane}

15. Immerse the gel in $0.25 \mathrm{M} \mathrm{HCl}$ during $10 \mathrm{~min}$ and rinse twice with distilled $\mathrm{H}_{2} \mathrm{O}$. Then, immerse the gel in denaturation solution during $30 \mathrm{~min}$ and rinse twice with distilled $\mathrm{H}_{2} \mathrm{O}$. Last, immerse the gel in $20 \times$ SCC during 5-15 min prior its transfer.

16. Transfer the gel in standard Southern-blot conditions using HybondXL hybridization nylon membrane (GE-Healthcare) in $20 \times$ SCC solution overnight. 
17. UV crosslink the DNA to the membrane by subjecting both sides to $70,000 \mu \mathrm{J} / \mathrm{cm}^{2}$ UV light using a $254 \mathrm{~nm}$ UV crosslinker.

18. Rinse the membranes with $2 \times$ SSC to eliminate salt excess and let them dry on a Whatman filter paper.

\subsubsection{Random primer radiolabeling}

19. Amplify a $218-$ pb fragment of the LEU2 gene by standard PCR with a Taq DNA polymerase and specific oligonucleotides (5'-GTTCCACT TCCAGATGAGGC- $3^{\prime}$ and $5^{\prime}$-TTAGCAAATTGTGGCTTGA-3'). Load the PCR product in an agarose gel, run an electrophoresis and purify the DNA from the agarose gel with a NucleoSpin Gel and PCR extraction kit following manufacturer instructions. To obtain an ultrapure probe, repeat the purification with the NucleoSpin Gel and PCR extraction kit following manufacturer instructions (PCR clean up protocol) and measure the final DNA concentration in the nanodrop.

20. Boil $200 \mathrm{ng}$ of the purified DNA probe in $36.5 \mu \mathrm{L}$ of milli-Q $\mathrm{H}_{2} \mathrm{O}$ $\left(100{ }^{\circ} \mathrm{C}\right.$ during $\left.10 \mathrm{~min}\right)$. Then, centrifuge the sample $(16,000 \mathrm{~g}, 30 \mathrm{~s})$ and quickly transfer the tube to ice.

21. Add $5 \mu \mathrm{L}$ of hexanucleotide mix, $5 \mu \mathrm{L}$ of $0.5 \mathrm{mM}$ dATG solution, $1 \mu \mathrm{L}$ of Klenow and $50 \mu \mathrm{Ci}$ of $32^{\mathrm{P}}$-dCTP. Mix by pipetting up and down and incubate for $1 \mathrm{~h}$ at $37^{\circ} \mathrm{C}$.

22. Purify from unincorporated labeled nucleotides through a Sephadex G50 column.

\subsubsection{Southern-blot hybridization}

23. Prehybridize the hybond-XL membrane in a hybridization bottle with $10 \mathrm{~mL}$ of Hybridization Solution under rotation in a hybridization oven at $65^{\circ} \mathrm{C}$ for at least $30 \mathrm{~min}$. Place the DNA side of the membrane facing inwards.

24. Add the radioactive probe (previously boiled during $10 \mathrm{~min}$ at $100^{\circ} \mathrm{C}$ for its denaturation) to $10 \mathrm{~mL}$ pre-warmed hybridization solution and incubate overnight under rotation in a hybridization oven at $65^{\circ} \mathrm{C}$.

25. Remove the radioactive solution and wash the filters twice during $5 \mathrm{~min}$ with $50 \mathrm{~mL}$ pre-warmed wash solution under rotation in a hybridization oven at $65^{\circ} \mathrm{C}$ and then perform an additional wash during $45 \mathrm{~min}$. Let the membranes dry on a Whatmann filter paper and detect the radioactive signals using a PhosphorImager Fujifilm FLA-5100. 


\section{Data analysis}

To determine the recombination frequency of a particular yeast strain, the average of at least three experiments, each performed with an independent transformant, should be calculated. From each transformant, we use the median value from six different colonies. Student's $t$-tests can be used to determine the statistical differences between the average recombination frequencies of different strains.

After the physical detection of the radioactive signals corresponding to the DSB and HR intermediates in the PhosphorImager Fujifilm FLA-5100, the ImageGauge software can be used for quantification. The exposition time in the PhosphorImager Fujifilm FLA-5100 should be optimized for each membrane to obtain non-saturated images. The analysis of the signals is performed by drawing quantification squares over each band (Fig. 1D). In addition, a second square of the same size is selected below each band to quantify the background from the same lane. Such signal of the background should be subtracted from the signal of each band in the gel. Then, the resulting signal corresponding to the each of the bands of the gel is used to estimate the percentage of molecules of interest at each time point of study. The percentage of molecules with DSBs is calculated by dividing the signal corresponding to the 2.4- and 1.4-kb bands by the sum of the signal of each all other bands (Fig. 1D and E). Similarly, the percentage of either molecules that constitute an intermediate for SCR or SCR + ICR reactions is calculated by dividing the signal corresponding to the 4.7- or 2.9-kb bands, respectively, by the sum of the signal of each all other bands (Fig. 1D and E).

\section{Acknowledgments}

Research in A.A.'s lab is funded by the Spanish Ministry of Science and Innovation, the European Research Council and the Junta de Andalucía, plus the European Union (FEDER).

\section{References}

Anand, R. P., Lovett, S. T., \& Haber, J. E. (2013). Break-induced DNA replication. Cold Spring Harbor Perspectives in Biology, 5(12), a010397. https://doi.org/10.1101/ cshperspect.a010397.

Cortés-Ledesma, F., \& Aguilera, A. (2006). Double-strand breaks arising by replication through a nick are repaired by cohesin-dependent sister-chromatid exchange. EMBO Reports, 7(9), 919-926. https://doi.org/10.1038/sj.embor.7400774. 
Cortes-Ledesma, F., Tous, C., \& Aguilera, A. (2007). Different genetic requirements for repair of replication-born double-strand breaks by sister-chromatid recombination and break-induced replication. Nucleic Acids Research, 35(19), 6560-6570. https://doi. org/10.1093/nar/gkm488.

Fasullo, M., Bennett, T., \& Dave, P. (1999). Expression of Saccharomyces cerevisiae MATa and MAT alpha enhances the HO endonuclease-stimulation of chromosomal rearrangements directed by his3 recombinational substrates. Mutation Research, 433(1), 33-44. https://doi.org/10.1016/S0921-8777(98)00059-7.

Fasullo, M. T., \& Davis, R. W. (1987). Recombinational substrates designed to study recombination between unique and repetitive sequences in vivo. Proceedings of the National Academy of Sciences of the United States of America, 84(17), 6215-6219. https://doi.org/ 10.1073/pnas.84.17.6215.

Gómez-González, B., \& Aguilera, A. (2019). Transcription-mediated replication hindrance: A major driver of genome instability. Genes and Development, 33(15-16). https://doi.org/10. 1101/gad.324517.119.

Gómez-González, B., Ruiz, J. F., \& Aguilera, A. (2011). Genetic and molecular analysis of mitotic recombination in Saccharomyces cerevisiae. In H. Tsubouchi (Ed.), DNA recombination. Methods in molecular biology (methods and protocols), vol 745 . Humana Press. https://doi.org/10.1007/978-1-61779-129-1_10.

Gonzalez-Barrera, S., Cortes-Ledesma, F., Wellinger, R. E., \& Aguilera, A. (2003). Equal sister chromatid exchange is a major mechanism of double-strand break repair in yeast. Molecular Cell, 11(6), 1661-1671. https://doi.org/10.1016/S1097-2765(03) 00183-7.

Heyer, W. D., Ehmsen, K. T., \& Liu, J. (2010). Regulation of homologous recombination in eukaryotes. Annual Review of Genetics, 44, 113-139. https://doi.org/10.1146/annurevgenet-051710-150955.

Johnson, R. D., \& Jasin, M. (2000). Sister chromatid gene conversion is a prominent double-strand break repair pathway in mammalian cells. The EMBO Journal, 19(13), 3398-3407. https://doi.org/10.1093/emboj/19.13.3398.

Kadyk, L. C., \& Hartwell, L. H. (1992). Sister chromatids are preferred over homologs as substrates for recombinational repair in Saccharomyces cerevisiae. Genetics, 132(2), 387-402.

Klein, H. L., Bačinskaja, G., Che, J., Cheblal, A., Elango, R., Epshtein, A., et al. (2019). Guidelines for DNA recombination and repair studies: Cellular assays of DNA repair pathways. Microbial Cell, 6(1). https://doi.org/10.15698/mic2019.01.664.

Lambert, S., \& Carr, A. M. (2013). Replication stress and genome rearrangements: Lessons from yeast models. Current Opinion in Genetics \& Development, 23(2), 132-139. https:// doi.org/10.1016/j.gde.2012.11.009.

Lee, J., Whang, I., \& Jayaram, M. (1996). Assembly and orientation of Flp recombinase active sites on two-, three- and four-armed DNA substrates: Implications for a recombination mechanism. Journal of Molecular Biology, 257(3), 532-549. https://doi.org/10.1006/jmbi. 1996.0183.

Mascioli, D. W., \& Haber, J. E. (1980). A cis-acting mutation within the MAT(a) locus of Saccharomyces cerevisiae that prevents efficient homothallic mating-type switching. Genetics, 94(2), 341-360.

Muñoz-Galván, S., Jimeno, S., Rothstein, R., \& Aguilera, A. (2013). Histone H3K56 acetylation, Rad52, and non-DNA repair factors control double-strand break repair choice with the sister chromatid. PLoS Genetics, 9(1), e1003237. https://doi.org/10. 1371/journal.pgen.1003237.

Nielsen, I., Bentsen, I. B., Lisby, M., Hansen, S., Mundbjerg, K., Andersen, A. H., et al. (2009). A Flp-nick system to study repair of a single protein-bound nick in vivo. Nature Methods, 6(10), 753-757. https://doi.org/10.1038/nmeth.1372. 
Ortega, P., Gómez-González, B., \& Aguilera, A. (2019). Rpd3L and Hda1 histone deacetylases facilitate repair of broken forks by promoting sister chromatid cohesion. Nature Communications, 10(1), 1-15. https://doi.org/10.1038/s41467-019-13210-5.

Pardo, B., Gómez-González, B., \& Aguilera, A. (2009). DNA repair in mammalian cells: DNA double-strand break repair: How to fix a broken relationship. Cellular and Molecular Life Sciences, 66(6), 1039-1056. https://doi.org/10.1007/s00018-009-8740-3.

Puget, N., Knowlton, M., \& Scully, R. (2005). Molecular analysis of sister chromatid recombination in mammalian cells. DNA Repair, 4(2), 149-161. https://doi.org/10.1016/ j.dnarep.2004.08.010.

Rudin, N., Sugarman, E., \& Haber, J. E. (1989). Genetic and physical analysis of double-strand break repair and recombination in Saccharomyces cerevisiae. Genetics, 122(3), 519-534.

Sadowski, P. D. (1995). The Flp recombinase of the 2- $\mu \mathrm{m}$ plasmid of Saccharomyces cerevisiae. Progress in Nucleic Acid Research and Molecular Biology, 51, 53-91. https://doi.org/10.1016/ S0079-6603(08)60876-4.

Tsalik, E. L., \& Gartenberg, M. R. (1998). Curing Saccharomyces cerevisiae of the 2 micron plasmid by targeted DNA damage. Yeast, 14(9), 847-852. https://doi.org/10.1002/ (SICI)1097-0061(19980630)14:9 < 847::AID-YEA285 > 3.0.CO;2-9.

Willis, N. A., \& Scully, R. (2021). Measurement of homologous recombination at stalled mammalian replication forks. In A. Aguilera, \& A. Carreira (Eds.), Methods in molecular biology: Vol. 2153. Homologous recombination. New York, NY: Humana. doi:10.1007/ 978-1-0716-0644-5_23.

Yeeles, J. T., Poli, J., Marians, K. J., \& Pasero, P. (2013). Rescuing stalled or damaged replication forks. Cold Spring Harbor Perspectives in Biology, 5(5), a012815. https://doi. org/10.1101/cshperspect.a012815. 